\title{
Physical Bounds on Implant Powering Efficiency Using Body-Conformal WPT Systems
}

\author{
Icaro V. Soares ${ }^{1}$, Mingxiang Gao ${ }^{2}$, Anja K. Skrivervik², Zvonimir Sipus 3 , Maxim Zhadobov ${ }^{1}$, \\ Ronan Sauleau ${ }^{1}$, Denys Nikolayev ${ }^{1}$ \\ ${ }^{1}$ IETR - UMR 6164, CNRS / Univ Rennes, France, ${ }^{2}$ MAG - SCI-STI-AS, EPFL, Switzerland, \\ ${ }^{3}$ FER, University of Zagreb, Croatia \\ denys.nikolayev@deniq.com
}

\begin{abstract}
The efficiency of an on-body wireless power transfer system for implant powering is defined by how the electromagnetic energy interacts with the lossy, heterogeneous, and dispersive body tissues. The objective of this study is to discuss the methodology and evaluate the theoretical bounds for the frequency-dependent electromagnetic energy transfer efficiency. We propose a simplified model that uses a finite tissueequivalent phantom enclosing an implantable receiver surrounded by a medium that represents a transmitter matched to the wave impedance of the body. This model is used to study different cases and evaluate the wireless power transfer efficiency as a function of the operating frequency and implantation depth. The obtained results can be used as a guideline to choose the design parameters and constraints of the on-body power source and gauge its performance against the predicted maximum achievable efficiency.
\end{abstract}

Keywords-biomedical electronics, implants, fundamental bounds, radiation efficiency, wireless power transfer.

\section{INTRODUCTION}

Wireless Power Transfer (WPT) has been a vastly investigated topic, and numerous techniques can be applied depending on the nature of the application [1]. For instance, this technology is particularly interesting for implantable bioelectronic and biosensor devices used to monitor physiological processes [2], stimulate body organs through electric signals [3], and drug delivery [4], among other possibilities. It enables faster and more precise diagnostics and treatments, in a less invasive and more secure way. However, WPT techniques that present a reasonable efficiency in most applications may exhibit a highly deteriorated performance when implantable devices are considered. To analyze and understand WPT in this context, it is required to consider the electromagnetic (EM) field behavior in dynamic, highly heterogeneous, dispersive, and lossy media as body tissues indeed are. Body-conformal phased surfaces are a promising practical approach to improve the WPT performance [5].

In order to properly evaluate the energy exchange between a transmitter source outside the body and the implanted receiver, one must model the EM wave propagation from the air to a dispersive body and how it affects the amount of transmitted power that reaches the receiver terminals. As stated in [6], three prominent factors affect the through-body EM energy transfer: 1) the attenuation of the EM waves in the lossy tissue (proportional to the operating frequency), 2) the wave-impedance mismatch at the boundaries between

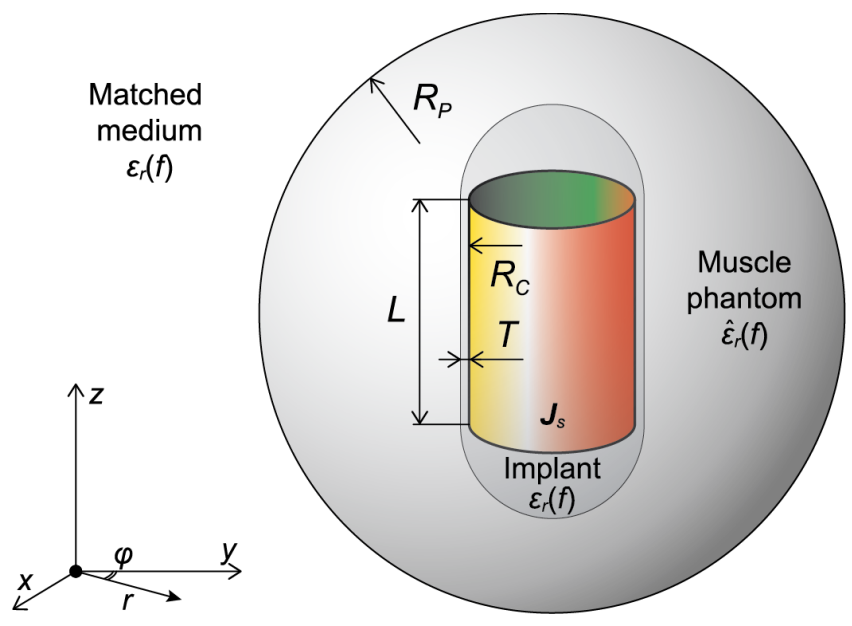

Fig. 1. Problem formulation (not to scale). The implant is centered inside of a spherical (radius $R_{P}$ ) muscle-equivalent phantom \{dispersive complex permittivity $\hat{\varepsilon}(f)$ according to [14]\}. The phantom is surrounded by a lossless medium with $\varepsilon_{r}(f)$. The receiving electric or magnetic antenna is modelled by corresponding current distributions $\boldsymbol{J}_{s}$ on a cylindrical aperture. The aperture is insulated by a lossless volume with $\varepsilon_{r}(f)$.

different media (especially, at the skin-air interface; can be partially mitigated [7]), and 3) the intrinsic efficiency decrease due to in-body antenna miniaturization (can be partially mitigated by dielectric loading [8]). Different modeling approaches have been proposed to assess the radiation efficiency and optimal frequency of implantable systems. The planar stratified model proposed in [9], [10], and various finite models were investigated by a spherical wave expansion in [11], [12] and by a full-wave approaches in [13].

This paper aims to develop a simple formulation for the electromagnetic energy exchange between an implantable receiver and an optimal on-body power source. In this way, we attempt to evaluate the best-case scenario of the achievable frequency-dependent energy transfer efficiency depending on the implantation depth and the receiver antenna type (magnetic or electric). Obtained results exceed the efficiencies of existing approaches thereby motivating further exploration and development of WPT solutions for powering of implants.

\section{Problem Formulation}

The goal is to analyze the achievable efficiency of wireless power transfer systems, which are composed of an implantable 
receiver antenna and a transmitter (Tx) radiating structure conformal to the body. Assuming that Tx antenna can be perfectly matched to the tissue environment (using high- $\varepsilon_{r}$ dielectrics, for instance [14]), this problem can be formulated through a simplified model shown in Fig. 1. In this model, the human body is represented by a homogeneous spherical phantom of radius $R_{p}$ having its complex dispersive permittivity $\hat{\varepsilon r}(f)$ set equivalent to the muscle tissue [15]. This phantom encloses an implantable receiver represented in Fig. 1 by a cylindrical surface with length $L$ and radius $R_{c}$, surrounded by a lossless capsule-shaped region representing an implant. Around the phantom (that is, external to the body) a lossless medium with its permittivity matched to muscle, represents the region of on-body transmitter. Note also that in [16] it was demonstrated that the shape of the body phantom only slightly influences the level of achievable power density just outside the body. The most important parameter is the distance between the implanted antenna and the surface of the body-position of the transmitter in our case.

Based on reciprocity [17], we consider fist an equivalent problem of the radiation source given by the electric current density $\boldsymbol{J}_{s}$ distribution of Fig. 1. The resulting radiated field leads to a power flow induced in the lossy phantom, and from the Poynting's theorem, the total energy is conserved as [17]:

$$
P_{t}=P_{r}+P_{d}+i 2 \omega\left(\overline{W_{m}}-\overline{W_{e}}\right),
$$

where $P_{t}, P_{r}$, and $P_{d}$ are the transmitted, received, and dissipated power densities, respectively, and $W_{m}$ and $W_{e}$ are the time-average magnetic and electric energies, respectively.

For the time-harmonic electric $\boldsymbol{E}$ and magnetic $\boldsymbol{H}$ fields, the source electric current density $\boldsymbol{J}_{\boldsymbol{s}}$, and the phantom's electric conductivity $\sigma$, the transmitted, received, and dissipated power can be calculated as follows:

$$
\begin{gathered}
P_{t}=\oint_{\Omega_{s}}\left(\frac{1}{2} \boldsymbol{E} \times \boldsymbol{H}^{*}\right) \cdot d \boldsymbol{s}, \\
P_{r}=\oint_{\Omega_{p}}\left(\frac{1}{2} \boldsymbol{E} \times \boldsymbol{H}^{*}\right) \cdot d \boldsymbol{s}, \\
P_{d}=\frac{1}{2} \int_{\Omega_{p}} \sigma|\boldsymbol{E}|^{2} d v .
\end{gathered}
$$

Note that the evaluation of ( $2 a-2 c)$ can be simplified by considering the problem's rotational symmetry. Therefore, the power transfer efficiency $\eta$ can be defined as:

$$
\eta \equiv \frac{\mathfrak{R}\left(P_{r}\right)}{\mathfrak{R}\left(P_{t}\right)}=\frac{1-\mathfrak{R}\left(P_{d}\right)}{\mathfrak{R}\left(P_{t}\right)} .
$$

As specified above, the effective radiation source is considered to be inside the phantom and the integration ( $2 b)$ is performed on the surface of the phantom. However, in the present analysis, the medium is linear and there is no polarization mismatch. Therefore, the reciprocity theorem can be applied [17], and the obtained results are valid for the original problem represented in Fig. 1. Hereafter, by a receiver

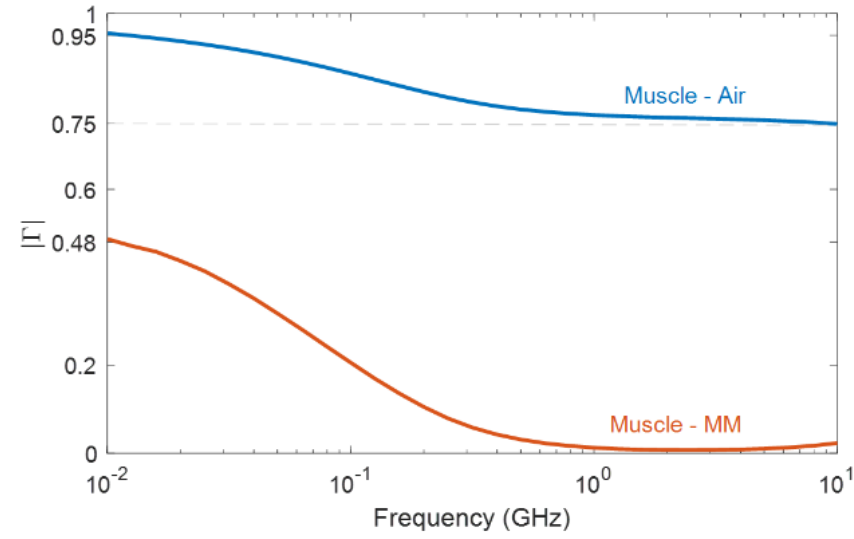

Fig. 2. Plane-wave reflection coefficients as a function of frequency for a muscle-air and a muscle-lossless-muscle (MM) interfaces.

antenna we refer to the one on the implant side. Accordingly, the transmitter implies the on-body WPT source.

The computation is made both for an electric and an a magnetic receiver antenna, represented respectively by the induced surface current densities $\boldsymbol{J}_{s}(r, \varphi, z)[13]$ :

$$
\begin{gathered}
\boldsymbol{J}_{s}^{E}=\left[0,0, \cos \left(\frac{\pi z}{L}\right)\right], \\
\boldsymbol{J}_{s}^{H}=[0,1,0] .
\end{gathered}
$$

In addition to the aforementioned assumptions, this model disregards the impedance mismatch and antennas and any other system-level losses (e.g., these of rectifiers). Moreover, a more realistic model would consider more complex heterogeneous geometry of the human body. However, the proposed model provides the efficiency evaluation of a wirelessly powered bioimplant device for the best-case scenario (i.e., the transmitter occupies the entire surface of the phantom, and the optimal energy confinement is achieved over the implant). The results obtained from this analysis are also useful as a guideline to define the frequency and what kind of source leads to the highest efficiency given the implant depth. Finally, by gauging the obtained bounds with the efficiency of a realistic on-body radiating structure (evaluated using the same phantom/receiver combination), one can gain an insight on the ways to further optimize the power transmitter system.

\section{RESULTS}

First, we show how matching the real permittivities of muscle and surrounding space (surrSp) affects the waveimpedance contrast on the tissue interface over a frequency range from $10 \mathrm{MHz}$ to $10 \mathrm{GHz}$. We use the reflection coefficient $\Gamma=\left(Z_{\text {muscle }}-Z_{\text {sursp }}\right) /\left(Z_{\text {muscle }}+Z_{\text {sursp }}\right)$ at the interface as a metric of contrast for demonstration purposes, where $Z_{n}=\left[j \omega \mu_{0} /\left(\sigma_{n}+j \omega \varepsilon_{n}\right)\right]^{1 / 2}$. As Fig. 2 shows, the reflection at the muscle-air boundary is inversely proportional to the frequency and varies from 0.95 to 0.75 . In the WPT context, such a contrast would lead to significantly reduced efficiency of the far-field and mid-field approaches. Conversely, when the 
intrinsic impedance of the transmitter is matched with the tissue [muscle-matched (MM) medium in our model; in practice, this can be realized using dielectric matching layers between the antenna and the body [18], [19]], the radiative energy is almost totally transmitted through the interface for frequencies of interest (roughly between $500 \mathrm{MHz}$ and $10 \mathrm{GHz}$; Fig. 2). Note that below $\sim 200 \mathrm{MHz}(\lambda>20 \mathrm{~cm}$ in muscle), the near-field $\mathrm{Tx} / \mathrm{Rx}$ coupling will occur, therefore we are not concerned with $\Gamma$ in $10-200-\mathrm{MHz}$ frequency range.

Next, we analyze the power transfer efficiency for the formulated model (Fig. 1). The model was implemented using COMSOL Multiphysics ${ }^{\circledR}$, and the power transfer efficiency was calculated using (2a-2c) and (3). The source size was $L=$ $1 \mathrm{~cm}$ and $R_{c}=L / 3$. The efficiency bounds for an electric $\eta_{E}$ and magnetic $\eta_{H}$ sources are evaluated as a function of the phantom radius (asymptotic to the implantation depth) and the frequency leading to the results in Fig. 3. The implantation depth ranges from $1 \mathrm{~cm}$ to $20 \mathrm{~cm}$, which embraces most of the potential applications except for the subcutaneous ones where an efficient inductive coupling can be implemented.

As shown in Fig. 3, a magnetic receiver outperforms the electric one for the near-field scenarios considered in this study. For the far-field region (starts around roughly $1-5 \mathrm{GHz}$ depending on the Rx depth; cf. Fig. 3), both receivers types show the same performance as only a propagating wave reaches them with no reactive components. The efficiency falloff in this region in governed by the attenuation. For the nearfield region, $\eta_{\mathrm{E}}$ sharply increases with the frequency before reaching its peak between roughly $1 \mathrm{GHz}$ and $3 \mathrm{GHz}$. On the other hand, $\eta_{\mathrm{H}}$ increases until reaching a plateau corresponding to $\max (\eta \mathrm{E})$ already at $\sim 100 \mathrm{MHz}$. Moreover, for either source, the efficiency is almost invariant with implantation depth for the frequencies $<100 \mathrm{MHz}$ due to a predominantly near-field operation.

\section{CONCLUSION}

The application of wireless power transfer techniques for biomedical implants opens a wide range of applications and possibilities. However, the power transfer performance is limited not only by the tissue losses but also by a difficulty of applying optimal design decisions in the absence of established theory. This study is an attempt to obtain the theoretical bounds for the efficiency of on-body power sources as a function of the operating frequency and implantation depth. Here, we assumed that an on-body power source is perfectly matched to the biological tissue and that an optimal focusing of energy is achieved from an aperture covering the entire surface of the phantom (based on the reciprocity and Tsymmetry principles).

The results obtained from this model showed that 1) magnetic receivers are preferable for the considered scenarios especially for the sub-GHz range of operating frequencies; 2 ) for WPT operating in far field $(f>1-5 \mathrm{GHz}$ depending of implantation depth), the receiver antenna type can be both electric or magnetic; 3) obtained results exceed the efficiencies of existing approaches thereby motivating further exploration and development of WPT solutions for powering of implants.

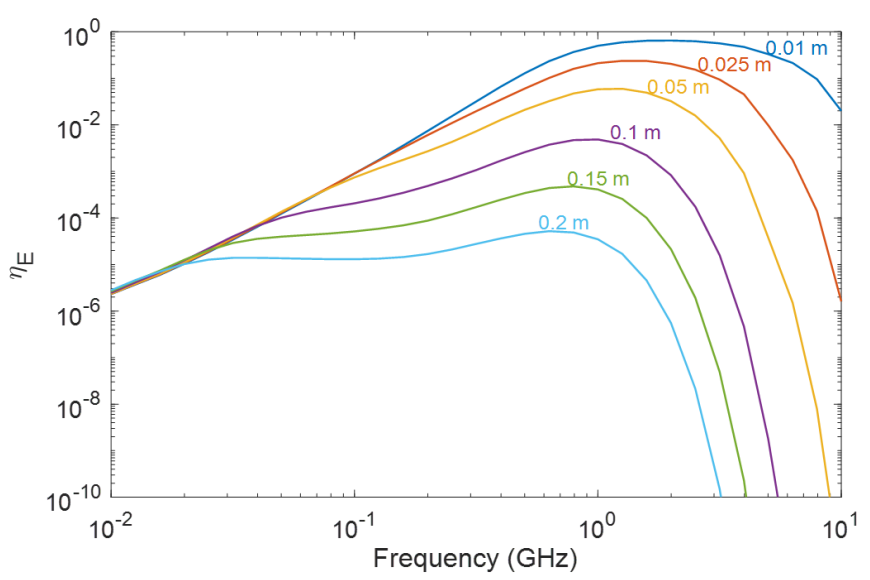

(a)

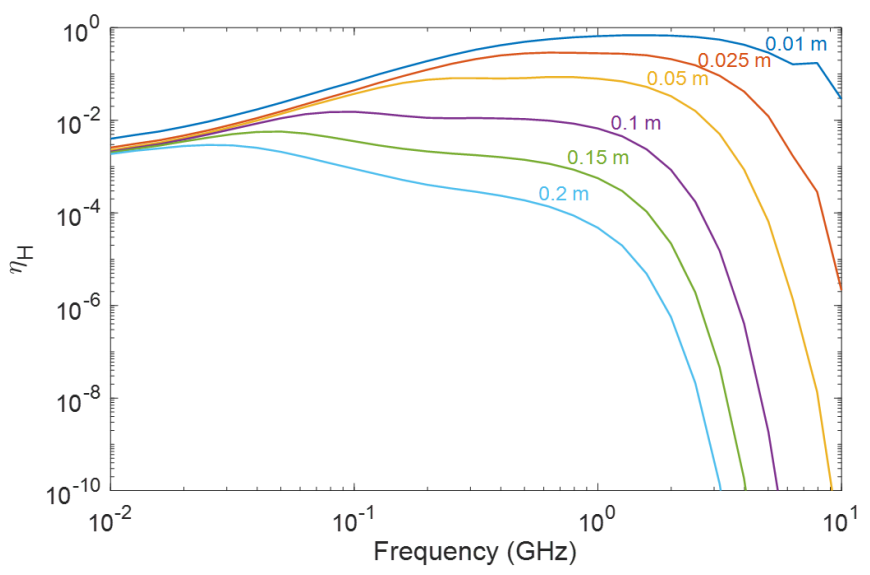

(b)

Fig. 3. Radiation efficiency for an (a) electric $\eta_{\mathrm{E}}$ and magnetic (b) $\eta_{\mathrm{H}}$ receiver as a function of the frequency and the phantom radius $R_{P}$ (asymptotic to the implantation depth).

Note that the results of this study contrast with the problem of implant radiation into a free space. There, an electric implantable source shows a higher peak efficiency than a magnetic one for a range of scenarios. This is indeed a fundamentally different problem as the implant radiation into free space implies interaction of the source with the tissue-air boundary and consequent re-radiation of induced surface currents (the reader is referred to [11], [13], [20] for more information on this topic).

To sum up, although this simplified model disregards many aspects of the real-life problem (highly heterogeneous medium, limitations on source design and its matching to tissues, sub-optimal focusing approaches, etc.), it provides a computationally fast and efficient guideline to analyze the best-case scenario operating conditions and thereby derive maximum expected wireless power transfer efficiency. Future work involves a gradual inclusion of various realistic aspects into the analysis.

\section{ACKNOWLEDGMENT}

This study was supported by the Région Bretagne through the project SAD "EM-NEURO". 


\section{REFERENCES}

[1] Z. Zhang, H. Pang, A. Georgiadis, and C. Cecati, "Wireless power transfer-An overview," IEEE Trans. Ind. Electron., vol. 66, no. 2, pp. 1044-1058, Feb. 2019.

[2] S. A. A. Shah and H. Yoo, "Radiative Near-Field Wireless Power Transfer to Scalp-Implantable Biotelemetric Device," IEEE Transactions on Microwave Theory and Techniques, vol. 68, no. 7, pp. 2944-2953, Jul. 2020.

[3] K. L. Kozielski, A. Jahanshahi, H. B. Gilbert, Y. Yu, Ö. Erin, D. Francisco, F. Alosaimi, Y. Temel, and M. Sitti, "Nonresonant powering of injectable nanoelectrodes enables wireless deep brain stimulation in freely moving mice," Science Advances, vol. 7, no. 3, p. eabc4189, Jan. 2021.

[4] H. Joo, Y. Lee, J. Kim, J.-S. Yoo, S. Yoo, S. Kim, A. K. Arya, S. Kim, S. H. Choi, N. Lu, H. S. Lee, S. Kim, S.-T. Lee, and D.-H. Kim, "Soft implantable drug delivery device integrated wirelessly with wearable devices to treat fatal seizures," Science Advances, vol. 7, no. 1, p. eabd4639, Jan. 2021.

[5] D. R. Agrawal, Y. Tanabe, D. Weng, A. Ma, S. Hsu, S.-Y. Liao, Z. Zhen, Z.-Y. Zhu, C. Sun, Z. Dong, F. Yang, H. F. Tse, A. S. Y. Poon, and J. S. Ho, "Conformal phased surfaces for wireless powering of bioelectronic microdevices," Nat. Biomed. Eng., vol. 1, p. 0043, Mar. 2017.

[6] D. Nikolayev, M. Zhadobov, P. Karban, and R. Sauleau, "Electromagnetic radiation efficiency of body-implanted devices," Phys. Rev. Applied, vol. 9, no. 2, p. 024033, Feb. 2018.

[7] F. Yang, P. M. Lee, Z. Dong, X. Tian, and J. S. Ho, "Enhancing wireless transmission from the body with wearable diffractive patterns," Phys. Rev. Applied, vol. 12, no. 5, p. 054020, Nov. 2019.

[8] D. Nikolayev, M. Zhadobov, and R. Sauleau, "Impact of tissue electromagnetic properties on radiation performance of in-body antennas," IEEE Antenn. Wireless Propag. Lett., vol. 17, no. 8, pp. 1440-1444, Aug. 2018.

[9] A. S. Y. Poon, S. O'Driscoll, and T. H. Meng, "Optimal frequency for wireless power transmission into dispersive tissue," IEEE Trans. Antennas Propag., vol. 58, no. 5, pp. 1739-1750, May 2010.
[10] J. S. Ho, S. Kim, and A. S. Y. Poon, "Midfield wireless powering for implantable systems," Proc. IEEE, vol. 101, no. 6, pp. 1369-1378, Jun. 2013.

[11] A. K. Skrivervik, M. Bosiljevac, and Z. Sipus, "Fundamental limits for implanted antennas: Maximum power density reaching free space," IEEE Trans. Antennas Propag., vol. 67, no. 8, pp. 4978-4988, Aug. 2019.

[12] M. Bosiljevac, Z. Sipus, and A. K. Skrivervik, "Propagation in finite lossy media: an application to WBAN," IEEE Antenn. Wireless Propag. Lett., vol. 14, pp. 1546-1549, Mar. 2015.

[13] D. Nikolayev, W. Joseph, M. Zhadobov, R. Sauleau, and L. Martens, "Optimal Radiation of Body-Implanted Capsules," Phys. Rev. Lett., vol. 122, no. 10, p. 108101, Mar. 2019.

[14] D. Nikolayev, W. Joseph, A. K. Skrivervik, M. Zhadobov, L. Martens, and R. Sauleau, "Dielectric-loaded conformal microstrip antennas for versatile in-body applications," IEEE Antenn. Wireless Propag. Lett., vol. 18, no. 12, pp. 2686-2690, Dec. 2019.

[15] S. Gabriel, R. W. Lau, and C. Gabriel, "The dielectric properties of biological tissues: III. Parametric models for the dielectric spectrum of tissues," Phys. Med. Biol., vol. 41, pp. 2271-2293, Nov. 1996.

[16] Z. Sipus, M. Bosiljevac, D. Nikolayev, and A. Skrivervik, "Design concerns for in-body antennas based on frequency analysis of fundamental radiation limitations," in Proc. 14th Eur. Conf. Antennas and Propagation (EuCAP 2020), Copenhagen, Denmark, 2020, pp. 1-5.

[17] J. D. Jackson, Classical Electrodynamics, 3rd ed. Hoboken, NJ: John Wiley \& Sons, 1999.

[18] S. Koulouridis, G. Kiziltas, Y. Zhou, D. J. Hansford, and J. L. Volakis, "Polymer-ceramic composites for microwave applications: fabrication and performance assessment," IEEE Transactions on Microwave Theory and Techniques, vol. 54, no. 12, pp. 4202-4208, Dec. 2006.

[19] J. Blauert and A. Kiourti, "Theoretical modeling and design guidelines for a new class of wearable bio-matched antennas," IEEE Trans. Antennas Propagat., vol. 68, no. 3, pp. 2040-2049, Mar. 2020.

[20] D. Nikolayev, Z. Sipus, M. Bosiljevac, W. Joseph, M. Zhadobov, R. Sauleau, L. Martens, and A. K. Skrivervik, "Optimal frequency of operation and radiation efficiency limitations of implantable antennas," in Proc. 14th Eur. Conf. Antennas and Propagation (EuCAP 2020), Copenhagen, Denmark, 2020, pp. 1-4. 